\title{
Refining Risk for Deep Vein Thrombosis in Hospitalized Patients
}

\author{
Anna L. Parks, $M D^{7}$ and R. Jeffrey Kohlwes, MD, MPH² \\ ${ }^{1}$ Internal Medicine Residency, University of California, San Francisco and Veterans Affairs Medical Center, San Francisco, CA, USA; ${ }^{2} \mathrm{PRIME}$ \\ Residency Program, University of California, San Francisco and Veterans Affairs Medical Center, San Francisco, CA, USA.
}

J Gen Intern Med 33(1):6

DOI: $10.1007 / \mathrm{s} 11606-017-4194-8$

(๑) Society of General Internal Medicine (outside the USA) 2017

$\mathrm{C}$ linicians often assume that the test characteristics for a diagnostic test are fixed. This allows clinicians to smoothly transition from a pre-test to a post-test probability when considering diagnostic or treatment thresholds. Unfortunately, these "fixed" test characteristics apply best to the derivation group studied and may not always be generalizable to other populations. Applying a diagnostic test outside a derivation population may lead to "spectrum bias," or the false assumption that a diagnostic test will work well in any population studied. ${ }^{1}$ An often-taught example of spectrum bias is the use of the outpatient-derived Wells score for the detection of deep venous thrombosis (DVT) in the inpatient setting. Compared to outpatients, hospitalized patients have a higher prevalence of pro-thrombotic characteristics such as immobility, comorbid illness and recent surgery, which makes the Wells score test characteristics less reliable in the inpatient setting.

The potential for spectrum bias leaves clinicians with few evidence-based options for assessing pre-test probability for DVT in hospitalized patients. The study by Alper et al. in this issue of JGIM puts forth a novel, inpatient-specific DVT risk stratification model. ${ }^{2}$ The authors collected data on risk factors for thromboembolism - including age, recent surgery or active cancer - among 2960 inpatients who were evaluated for DVT with lower extremity ultrasound at an academic quaternarycare hospital. They then generated four clinical characteristics that were associated with ultrasound-diagnosed DVT, and assigned each characteristic a point value based on odds ratios: prior DVT (6 points), active cancer (1 point), hospital stay $\geq 6$ days ( 1 point), and age $\geq 46$ years ( 1 point). These points were added to generate a CEBI [Center for Evidence-Based Imaging] score that categorized patients as low-risk ( 0 points) or high-risk ( $\geq 1$ points) for DVT. The CEBI score demonstrated high sensitivity (98.1\%) and negative predictive value
(97.1) and adequate AUC (0.73) for discerning which hospitalized patients were at risk for proximal DVT.

The CEBI scoring system improves substantially on the Wells Score, which performs poorly in inpatients. As the authors rightly point out, its strength is in accurately identifying low-risk patients who do not require further imaging, potentially reducing overuse. Conversely, another key point to emerge from this data is that our threshold for obtaining an ultrasound should be quite low. Just one point on the CEBI score - for example, any patient aged 46 or older - defines a patient as high-risk. More than $90 \%$ of patients in this study met that criterion, and over one in five of these high-risk patients had a DVT. Ultrasound is relatively inexpensive, non-invasive and widely available, and does not expose patients to radiation. Moreover, venous thromboembolism is associated with significant morbidity and mortality but can be safely treated in most patients. With all this in mind, we can now feel more confident in deferring ultrasound in truly lowrisk patients, but we should not hesitate to pursue diagnostic imaging in the majority of hospitalized patients. The only other caveat to this study is that it should be confirmed in other, non-tertiary-care hospitals, to ensure that the test characteristics hold up across the spectrum of inpatient care.

Corresponding Author: R. Jeffrey Kohlwes, MD, MPH; PRIME Residency Program University of California, San Francisco and Veterans Affairs Medical Center, San Francisco, CA, USA (e-mail: jeff.kohlwes@ucsf.edu).

\section{Compliance with Ethical Standards:}

Conflict of Interest: The authors declare that they do not have a conflict of interest.

\section{REFERENCES}

1. Mulherin SA, Miller WC. Spectrum Bias or Spectrum Effect? Subgroup Variation in Diagnostic Test Evaluation. Ann Intern Med. 2002;137(7):598602.

2. Alper EC, Ip IK, Silveira PC, et al. Risk stratification model: lower extremity ultrasonography for hospitalized patients suspected of deep vein thrombosis. J Gen Intern Med. https://doi.org/10.1007/s11606-017-4170-3. 